\title{
GENERAL MASSLESS FIELD EQUATIONS FOR HIGHER SPIN IN DIMENSION 4
}

\author{
Roman Lavicka ${ }^{1}$, vladimir soucek ${ }^{2}$, and Wei Wang ${ }^{3}$ \\ ${ }^{1}$ Charles University \\ ${ }^{2}$ Charles University, Prague \\ ${ }^{3}$ Zhejiang University
}

September 27, 2020

\begin{abstract}
Massless field equations are fundamental in particle physics. In Clifford analysis, the Euclidean version of these equations has been dealt with but it is not clear, even in dimension 4, what should be the right analogue of massless field equations for fields with values in a general irreducible Spin(4)-module. The main aim of the paper is to explain that a good possibility is to take the so-called generalized Cauchy-Riemann equations proposed a long time ago by E. Stein and G. Weiss. For this choice of the equations, we show that their polynomial solutions form different irreducible Spin(4)-modules. This is an important step in developing the corrresponding function theory.
\end{abstract}

\section{Hosted file}

masslessFE7.pdf available at https://authorea.com/users/362231/articles/483452-generalmassless-field-equations-for-higher-spin-in-dimension-4 